\title{
Formation of artificial lipid bilayers using droplet dielectrophoresis $\uparrow$
}

\author{
Sara Aghdaei, Mairi E. Sandison, Michele Zagnoni, Nicolas G. Green and Hywel Morgan* \\ Received 30th April 2008, Accepted 5th August 2008 \\ First published as an Advance Article on the web 13th August 2008 \\ DOI: $10.1039 / b 807374 k$
}

We describe the formation of artificial bilayer lipid membranes (BLMs) by the controlled, electrical manipulation of aqueous droplets immersed in a lipid-alkane solution. Droplet movement was generated using dielectrophoresis on planar microelectrodes covered in a thin insulator. Droplets, surrounded by lipid monolayers, were brought into contact and spontaneously formed a BLM. The method produced BLMs suitable for single-channel recording of membrane protein activity and the technique can be extended to create programmable BLM arrays and networks.

Artificial BLM techniques are of great importance in membrane protein research. Electrophysiological studies of proteins reconstituted into BLMs can generate detailed information at the single molecule level on protein activity, ligand-binding and kinetics. ${ }^{1}$ Classical BLM techniques have been in use for over 40 years, but are not amenable to applications where high-throughput processes and reproducibility are required (e.g. drug screening and biosensing applications). ${ }^{2}$ New labon-chip technologies are being developed which allow for ever greater integration of complex functions with precise control of fluids on the micro-scale. ${ }^{3}$ This technology can be exploited to combine parallelization, achieved through microfluidics, with the sensitivity and selectivity of BLM approaches. Although various miniaturized devices have been reported, only a few BLM formation methods ${ }^{4-7}$ have been developed on-chip that are reproducible, potentially automatable and suitable for singlechannel recording.

An alternative technique was recently described by Holden et al., ${ }^{8-12}$ which uses an alkane-lipid solution as the bulk phase, as opposed to an aqueous electrolyte. Droplets of buffer are immersed in the organic solution and at the interface between the two phases lipid monolayers form. When two droplets are brought into contact, a BLM forms at the interface between the droplets. As well as forming single BLMs, this approach allows for the creation of BLM networks. However, the method required manual manipulation of the droplets using micromanipulators.

Electrical methods for droplet manipulation exist, based on electrodynamic techniques, such as dielectrophoresis (DEP) and electro-wetting on dielectric (EWOD). ${ }^{13,14}$ Droplet move-

School of Electronics and Computer Science, University of Southampton, Southampton, UK; Fax: +44 2380 593029; Tel: +44 2380593330.

E-mail:hm@ecs.soton.ac.uk

$\dagger$ Electronic supplementary information (ESI) available: Supplementary information, supplementary Fig. S1-S3 and video legends, videos 1-3. See DOI: $10.1039 / \mathrm{b} 807374 \mathrm{k}$ ment occurs because of differences in the polarisability of two dielectrics which produces an interfacial stress leading to droplet deformation. In a non-uniform electric field, the droplet can move towards or away from regions of high electric field depending on the polarisability difference. ${ }^{9}$ There are a number of advantages to electrical droplet actuation: it enables precise control of nano- and picoliter droplets (facilitating displacement, formation, division and mixing), is programmable and easily integrated into lab-on-a-chip devices. ${ }^{15,16}$

Here, we describe the application of electrodynamic techniques to the formation of artificial BLMs by the manipulation, via an electronic switching circuit, of aqueous droplets immersed in a decane-lipid phase. This method is simple and amenable to automation and to integration with other fluidic functions, as well as to the development of a flexible, reconfigurable dropletbased BLM array.

The device is shown in Fig. 1(a). It consists of six pairs of individually addressable, thin-film, platinum electrodes, patterned using standard photolithographic techniques (ESI $\dagger$ ) on a glass substrate. The electrodes were coated with a $0.7 \mu \mathrm{m}$ insulating layer of SU8. A low-frequency electric field is applied between two or more planar micro-electrodes, with the dielectric layer preventing electrochemical reactions. A plastic reservoir was bonded to the surface of the device to contain the lipid-decane solution and the electrodes were connected to an AC frequency generator and amplifier via an electronic switching circuit. BLM capacitance and transmembrane currents were measured using a pair of $\mathrm{Ag} \mid \mathrm{AgCl}$ electrodes inserted into the droplets and connected to a BLM amplifier. An upright microscope was employed to visualize the droplets.

In order to control droplet movement, two different electrode geometries were incorporated into the design. The outer electrode pairs (1-1' and $44^{\prime}$ in Fig. 1(b)) were wedge-shaped, with the gap between a pair narrowing towards the centre of the device. This meant that droplets at the outer edges of these electrodes moved inwards towards the two smaller, inner electrode pairs $\left(2-2^{\prime}\right.$ and $\left.3-3^{\prime}\right)$ when a potential was applied, as the electric field strength increased towards the centre of the device. Two smaller electrode pairs (2-2' and 3-3') were situated at the centre. When a potential was applied only to these inner electrodes, the region of highest field strength was located at the centre of the device; applying a potential to both the inner and outer electrode pairs produced high field regions between the pairs (Maxwell stress tensor simulations in the ESI and Fig. S1†). These two electric field configurations were used to form a BLM as follows (Fig. 1(b)-(e)).

After filling the fluid reservoir with $20 \mathrm{mg} \mathrm{ml}^{-1}$ asolectin in $n$-decane, two $2 \mu \mathrm{L}$ droplets of $100 \mathrm{mM} \mathrm{KCl}$ were placed onto opposite outer electrodes. The positioning of these droplets is 


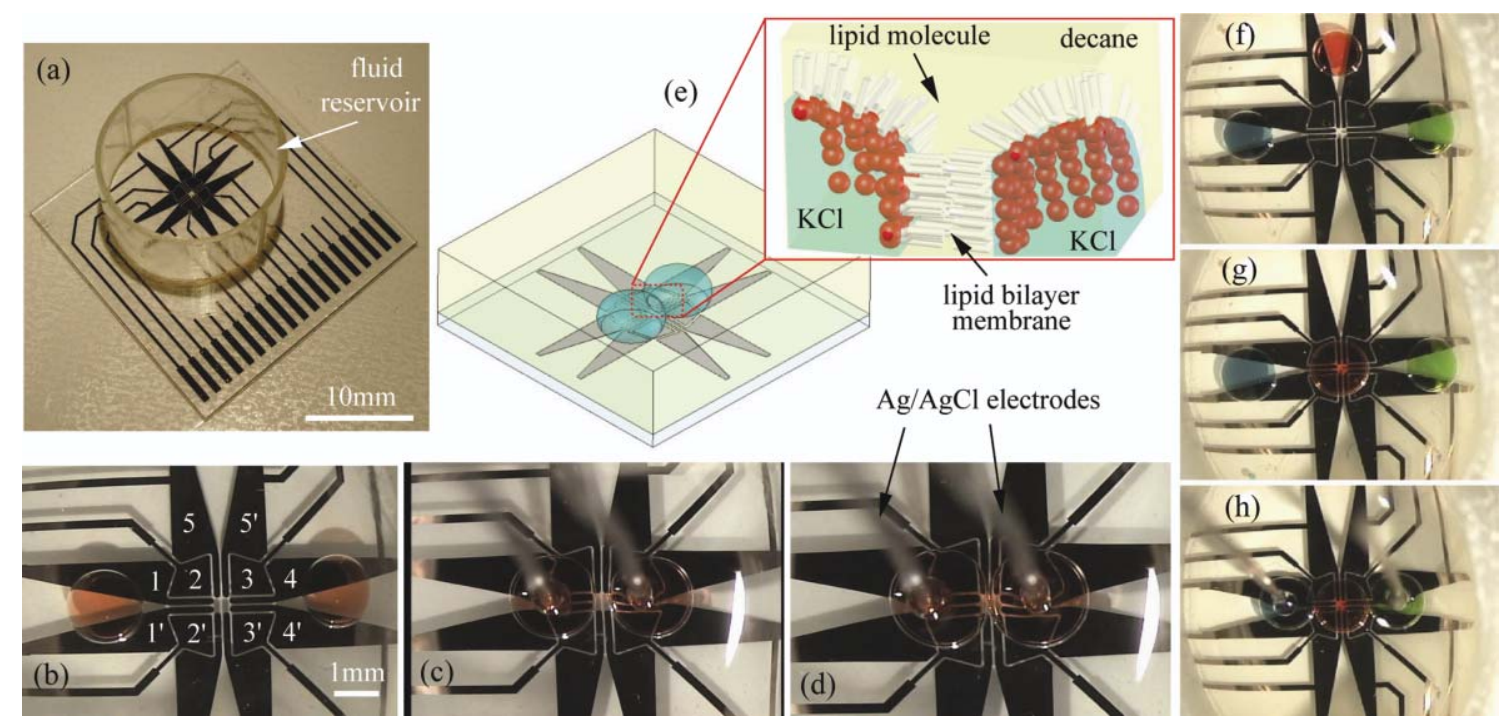

Fig. 1 Droplet-interface BLMs. (a) Device for producing BLMs using DEP. (b) Droplets are initially dispensed onto the outer edges of two electrode pairs, $1-1^{\prime}$ and $4-4^{\prime}$ (the droplets in the images contain food dye in order to better visualize them). (c) A potential is applied between these outer electrodes and the inner electrodes to move the droplets towards the centre. (d) To bring the droplets into contact, a potential is applied to the inner electrodes (2-2' and 3-3') only. (e) A BLM is formed by the two contacting lipid monolayers at the droplet-droplet interface. (f)-(h) Formation of a three-droplet BLM network.

not critical -DEP pulls them onto the electrodes, the droplet symmetrically between the pair of electrodes. In this work we routinely used $2 \mu \mathrm{L}$ volume droplets, but the volume that can be manipulated depends on the size of the microfabricated electrodes. Further work is required to determine this relationship and quantify the smallest volume that can be moved. The droplets were left to stabilize for 2-3 minutes, during which time a monolayer of phospholipids self-assembled at the interfaces between the two phases. The two droplets were then moved towards the regions between the inner and outer electrodes, by applying $12 \mathrm{~V}$ at $2 \mathrm{kHz}$ to both electrode pairs, giving a typical droplet velocity of a few $\mathrm{mm} \mathrm{s}^{-1}$ (details in the ESI $\dagger$ ). At this point, $\mathrm{Ag} \mid \mathrm{AgCl}$ electrodes, whose sharpened ends were coated with a thin layer of agarose gel, were inserted into each droplet from above, and then fixed in position. These electrodes did not hinder the movement of the droplet and could be inserted and removed without affecting the droplets in any way. A potential of $3 \mathrm{~V}$ at $2 \mathrm{kHz}$ was then applied to the inner electrode pairs only, in order to slowly move the droplets to the centre and into contact (ESI video $1 \dagger$ ), after which the applied voltage was turned off.

BLM formation was monitored by measuring the capacitance between the two droplets. This was done using the $\mathrm{Ag} \mid \mathrm{AgCl}$ electrodes connected to a high sensitivity ID562 BLM amplifier (Industrial Developments Bangor, Bangor, UK), by applying a $20 \mathrm{mV}$ triangular waveform. A typical trace is presented in Fig. 2(a), which shows that upon droplet contact the capacitance rises, increasing in two stages until a stable value is reached. Initially the contact area is very small (small capacitance), but it grows with time until an equilibrium is established. The reason for the two separate stages in the growth of the bilayer is not yet clear, but experimental evidence (measurement of Gramicidin ion channel currents) confirms that bilayers were present even at very low capacitance values. The time required to reach a stable capacitance value varied, depending upon variations in droplet volume and the time from droplet injection to BLM formation, and a mean capacitance of $335 \pm 180 \mathrm{pF}$ was measured $(n=$ 15 , error of one standard deviation). Assuming a specific membrane capacitance of approximately $0.5 \mu \mathrm{F} \mathrm{cm}^{-2},{ }^{17}$ a BLM diameter of $283 \pm 74 \mu \mathrm{m}$ is obtained. The BLMs were found to be stable, with a maximum lifetime of approximately $20 \mathrm{~h}$ (ESI Fig. S2 $\dagger$ ). This BLM formation technique was extremely reliable: over the course of $>100$ trials, a BLM was produced on every attempt.

In addition to forming BLMs by electrodynamic droplet control, the separation of droplets and the subsequent re-formation of a BLM could be performed repeatedly (ESI video $2 \dagger$ ). By applying a potential $(3 \mathrm{~V}$ at $2 \mathrm{kHz})$ to the outer electrode pairs only, two BLM-forming droplets could be pulled apart and a new BLM then formed as described previously. This process is illustrated in Fig. 2(b), where the variation of capacitance with droplet position is shown. The data in this figure shows disassembly of a bilayer with relatively low capacitance (35$40 \mathrm{pF}$ ), but it was equally possible to disassemble bilayers with much higher values of capacitance. Being able to disassemble and reform BLMs in this manner means that, if a number of droplets containing different buffers or proteins were dispensed onto a device, a controlled sequence of BLMs with diverse environments could be readily created.

In order to assess the suitability of the BLMs for performing electrophysiological studies on membrane proteins, the breakdown voltage of a BLM was measured, giving a mean value of $290 \pm 30 \mathrm{mV}$. This value is similar to that achieved with classical BLM apparatus. At this voltage, the BLM broke and the droplets merged and mixed (ESI video $3 \dagger$ ). In addition, single channel recordings were acquired by mixing gramicidin $\mathrm{D}(10$ $20 \mathrm{pg} \mathrm{m}^{-1}$ ) into the droplet buffer prior to BLM formation. Gramicidin monomers diffuse in opposite leaflets of a lipid bilayer and transiently assemble into BLM-spanning dimers 

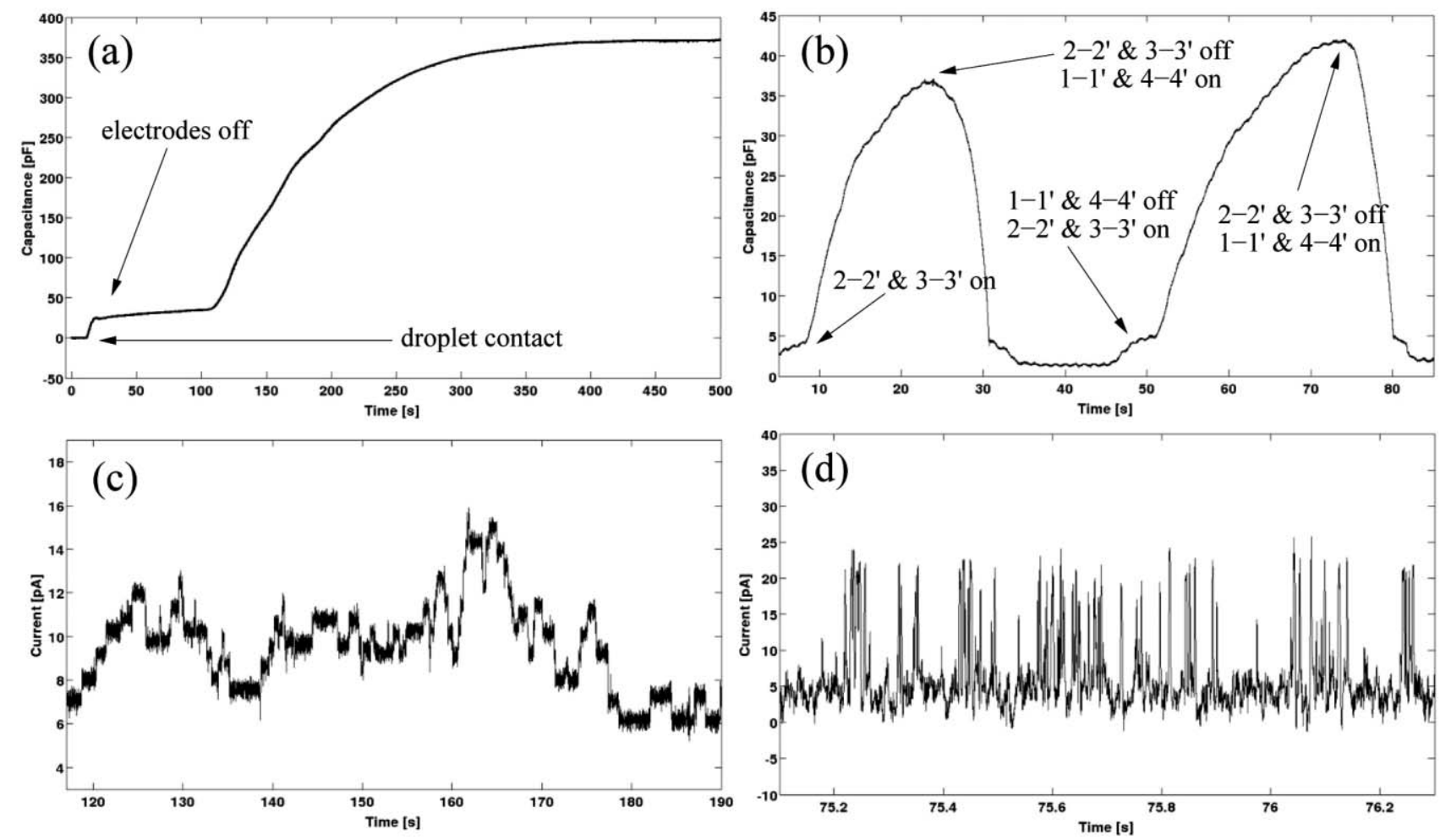

Fig. 2 Electrical recordings of BLM formation and ion-channel conduction. (a) A capacitance trace acquired during BLM formation. The capacitance rises from the baseline following droplet contact. (b) The formation and disassembly of a droplet-interface BLM, repeated twice. A potential was applied to the inner electrodes (2-2' and 3-3') to form a BLM and to the outer electrodes (1-1'and 4-4') to pull the droplets apart. (c) A trace showing ion-conduction through gramicidin channels. (d) A recording of single-channel KcsA activity. Both ion-channel recordings were acquired at $100 \mathrm{mV}$.

forming ion channels. ${ }^{18} \mathrm{~A}$ trace showing ion conduction through gramicidin channels in a droplet BLM is presented in Fig. 2(c).

Larger membrane proteins were also inserted into a droplet BLM. Fig. 2(d) shows a recording of activity from the $\mathrm{K}^{+}$ channel KcsA (MW $67 \mathrm{kDa}$ for the tetrameric form), which was delivered to a BLM formed using 1: 1 POPG : POPC (Avanti Polar Lipids, USA). Note that changing the lipid composition (tested with POPG, POPC, DPhPC and asolectin) had no observable effect on the formation or stability of the bilayer. Lipid vesicles (150-350 nm diameter) containing KcsA (purified and reconstituted as described previously) ${ }^{19}$ were mixed into one of the droplet buffers. This buffer had a high salt concentration $(600 \mathrm{mM} \mathrm{KCl})$ with $\mathrm{pH}=7.4$. Because the $\mathrm{Kcs} A$ channel opens only in an acidic environment (in the presence of anionic lipids such as POPG), the second droplet buffer (150 mM KCl) had a $\mathrm{pH}$ of $4 .^{20}$ Following droplet contact, a salt gradient was established across the droplet-interface BLM initiating vesicle fusion and leading to observable KcsA electrical activity (the conductance level and open probability of the channel is similar to that observed previously). ${ }^{19,20}$

A network of BLMs could also be formed. The formation of two BLMs at the interfaces between three droplets is illustrated in Figs. 1(f)-(h) (protocol in ESI $\dagger$ ). All three droplets contained $100 \mathrm{mM} \mathrm{KCl}$, but the central droplet also contained $10 \mathrm{pg} \mathrm{m}^{-1}$ gramicidin D. The formation of two BLMs was confirmed by recording ion-channel activity of gramicidin, which incorpo- rated into both BLMs from the central droplet. When one or more gramicidin channels formed in both BLMs simultaneously, a current was measured between the outer droplets (ESI Fig. S3†).

In conclusion, we have shown that DEP control of droplets provides a flexible method for forming BLMs within a microsystem under simple electronic control at low electrical potentials $(\sim 10 \mathrm{~V})$. The BLMs have long lifetimes and are suitable for the acquisition of electrophysiological data from membrane proteins. Although the $\mathrm{Ag} \mid \mathrm{AgCl}$ current recording electrodes are inserted by hand, future systems could integrate these electrodes onto a single platform. The approach described in this paper is particularly suited to the development of programmable biological assays, integrated with micro-chip fluidic operations, and could be used for large-scale, automated, microarray platforms for membrane protein analysis.

\section{Acknowledgements}

This work was supported by the UK Interdisciplinary Research Centre in Bio-Nanotechnology (R45659/01) and the $6^{\text {th }}$ Framework Program of the European Commission under contract NMP4-CT-2005-017114 "RECEPTRONICS". Vesicles containing KcsA were a kind gift from Dr Phedra Marius and Prof. Anthony Lee (School of Biological Sciences, University of Southampton, UK). 


\section{Notes and references}

1 Y. Jiang et al., Nature, 2002, 417, 515-522.

2 A. P. F. Turner and N. Magan, Nat. Rev. Microbiol., 2002, 2, 161-166.

3 J. Atencia and D. J. Beebe, Nature, 2005, 437, 648-655.

4 M. Andersson et al., Langmuir, 2007, 23, 2924-2927.

5 M. E. Sandison, M. Zagnoni and H. Morgan, Langmuir, 2007, 23, $8277-8284$.

6 B. Le Pioufle, H. Suzuki, K. V. Tabata, H. Noji and S. Takeuchi, Anal. Chem., 2008, 80, 328-332.

7 M. Kreir, C. Farre, M. Beckler, M. George and N. Fertig, Lab Chip, 2008, 8, 587-595.

8 M. A. Holden, D. Needham and H. Bayley, J. Am. Chem. Soc., 2007, 129, 8650-8655.

9 W. L. Hwang, M. A. Holden, White and H. Bayley, J. Am. Chem. Soc., 2007, 129, 11854-11864.

10 J. R. Thompson, A. J. Heron, Y. Santoso and M. I. Wallace, Nano Lett., 2007, 7, 3875-3878.
11 A. J. Heron, J. R. Thompson, A. E. Mason and M. I. Wallace, J. Am. Chem. Soc., 2007, 129, 16042-16047.

12 W. L. Hwang, M. Chen, B. Cronin, M. A. Holden and H. Bayley, J. Am. Chem. Soc., 2008, 130, 5878-5879.

13 P. Gascoyne et al., Lab Chip, 2004, 4, 299-309.

14 F. Mugele, J. C. Baret and J. Phys, Condens. Matter, 2005, 17, R705R774.

15 R. B. Fair, Microfluid. Nanofluid., 2007, 3, 245-281.

16 S. K. Cho, Y. Zhao and C. J. Kim, Lab Chip, 2007, 7, 490498.

17 Y. Cheng, R. J. Bushby, S. D. Evans, P. F. Knowles, R. E. Miles and S. D. Ogier, Langmuir, 2001, 17, 12401242.

18 B. M. Burkhart, R. M. Gassman, D. A. Langs, W. A. Pangborn and W. L. Duax, Biophys. J., 1998, 75, 2135-2146.

19 P. Marius et al., Biophys. J., 2008, 94, 1689-1698.

20 M. Zagnoni, M. E. Sandison, P. Marius, A. G. Lee and H. Morgan, Lab Chip, 2007, 9, 1176-1183. 